\title{
Propagation of Excitation Waves and Their Mutual Interactions in the Surface Layer of the Ball with Fast Accessory Paths and the Pacemaker
}

\author{
Jiří Kroc \\ West Bohemia University, Research Center, Univezitní 8, \\ CZ 332 03, Plzeň, Czech Republic, \\ krocj@ntc.zcu.cz,

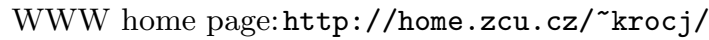

\begin{abstract}
Propagation of excitation waves and their mutual interactions on the surface layer of a ball with fast accessory paths and pacemaker is studied by the cellular automaton (CA) computer simulation technique in three-dimensions. The existence of the conducting system created by fast accessory paths and the pacemaker in the excitable medium - i.e., in the medium having a morphology - is important for this study. The topology of the medium and the CA-model itself are kept quite simple in order to focus the main attention of this study into the spatio-temporal response of the excitable medium with respect to the position and size of excitatory disturbances and inclusions. Propagation of undistorted excitation waves in the homogeneous excitable medium and appearance of spirals in it - induced by initial excitatory disturbances are studied as well.
\end{abstract}

\section{Introduction}

Propagation of excitatory process through the human heart [6] is a quite complex problem that deserves a great importance in non-invasive diagnosis of heart diseases. It should be emphasized that this complexity is manifold. The general aim of this article is to elucidate some parts of this complexity using a simple model and a simple topology.

The excitation of a myocardial cell leads to changes of the trans-membrane potential that triggers the action potential (AP) propagating parallel to the surface of the myocardial cell $[7,8]$. This results in activation of neighbouring cells via electrotonic currents across the cell junctions. It is quite well known from experimental data that pathological changes of the heart, e.g. ischemia, dysplasia, etc., can change the shape of AP and/or coupling between cells. Macroscopically this leads to changes of the propagation of activation waves across the muscle tissue.

The model proposed in this paper contains several following simplifications. It works above the level of living cells. The simplified topology is used, i.e. a surface 
layer of a ball with two fast accessory paths (the conducting system), and sinoatrial (SA) node that serves as the pacemaker. Muscle fibres - and anisotropy of excitation propagation resulting from it - are not taken into account.

It seems to be quite interesting to study development and interactions of propagating excitation fronts in three-dimensional (3D) excitable medium having a simple conducting system. Certain number of computational studies of spiral development and its mandering is known in the plane without the conducting system $[4,5]$ but no one with the simple conducting system to the best knowledge of the author. The conducting system is the element in excitable media that can brings new types of responses and effects. Therefore, it deserves a computational study.

CA-model discretize space into three-dimensional (3D) lattice of cubes. Cubes, i.e. elements of this lattice, are called cells. Every cell has defined a neighbourhood - usually a list of nearest neighbouring cells and the cell itself that is uniform through the whole lattice. Every cell contains a list of variables, e.g. state, morphology, etc. Morphology is divided into three different classes: conducting system, muscle, and the SA-node. The evolution of the system is driven by a transition rule that computes new values of variables using values of cells laying in the neighbourhood of given cell from the previous CA-step. General information about the CA simulation technique with applications can be found in the book [1], in the articles of Vichniac [12], Toffoli [10] and in books $[11,13]$.

The topology of medium and the CA-model itself as mentioned before are kept quite simple in order to focus the main attention of this study into the spatio-temporal response of the excitable medium with respect to the position and size of excitatory disturbances and inclusions - having slower excitation. Propagation of undistorted excitation waves in the homogeneous excitable medium with the conducting system and appearance of spirals in it - induced by initial excitatory disturbances - are studied as well.

A new simulation cellular automata environment has been developed - as a part of this study that is important for future simulations - to simulate propagation of excitation in excitable media. This code is written in $C$ and cooperates with the OpenGL graphical library [9] and runs in X-Windows window system under Linux. The OpenGL enables us, beside the other advantages, to display data using transparentness.

\section{Cellular Automaton Model of Propagation of Excitation Waves}

The proposed CA-model works with the uniform 3D-lattice of cube cells that are updated simultaneously according to a transition rule. As mentioned above, the evolution of states of one particular cell is controlled by the set of cells that form the neighbourhood or surrounding of this cell. In this model, the so called Moore neighbourhood, i.e. the twenty-six first and second nearest neighbours of an updated cell and this updated cell itself is used. The proposed probabilistic 
excitation wave front movement leads to approximately isotropic propagation of this front. The lattice size used is the cube composed of $200^{3}$ cells - i.e., $8 \times 10^{6}$ cells in total - for large simulations, and $100^{3}$ cells for small simulations. Smaller lattice size requires substantially smaller simulation time.

Generally, the transition rule can be split into several sequential steps that handle the evolution of different parts of the cellular automaton belonging into different morphological classes, i.e. the conducting system, muscle, and the SAnode. Due to computational reasons, one additional morphological class is defined with the empty value that should be understood in the following sense. A cell having the empty morphological state is not included into propagation of excitation events but is necessary because this CA-model works with the neighbourhood that strictly requires all neighbouring cells. Every surface cell has at least one cell with empty morphological state in its neighbourhood.
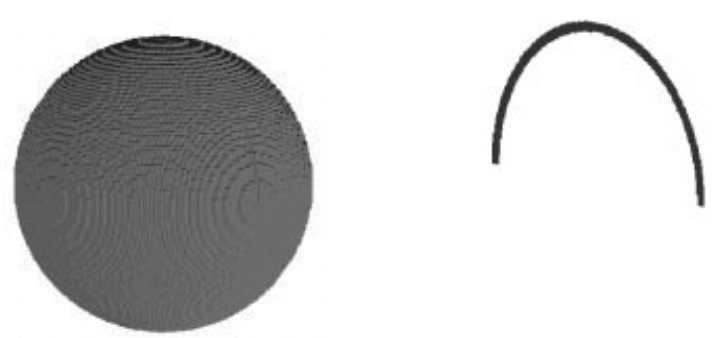

Fig. 1. The excitable medium is created by the ball surface layer, cf. the left side of the figure. Two fast accessory paths with the SA-node in between of them creates the conducting system, cf. the right side of the figure.

Each cell in the CA-model has two variables, namely, state and morphology. Variable state of the cell defines the level of excitation of the cell laying in the interval of $\langle 0,21\rangle$. State equal to the value of 21 is the excited state. As the value of the state variable decreases, first absolutely refractory states and then relatively refractory states are reached. The resting state, i.e. the situation when the cell waits for an excitation event, has assigned the zero value. Every cell inside of the lattice belongs to one of the following morphology classes, i.e. empty, the conducting system, muscle, and the $\mathrm{SA}$-node with values of 0,1 , 2 , and 3, respectively. All cells with values between 1 and 3 defines excitable medium. Cells with value equal to zero, i.e. empty cells, does not influence the excitation process at all.

It is assumed in this model that the excitable medium is a ball surface layer ${ }^{1}$ with two branches of conducting system laying in it, cf. Fig. 1. The SA-node

\footnotetext{
${ }^{1}$ The diameter of this ball is slightly below 200 cubes. Due to this reason, all figures presented in this work have a big granularity because sides of those cubes are visible.
} 
serving as the pacemaker is located at the top of the ball and at the same time in the between of two branches of the conducting system. Excitation is generated by the $\mathrm{SA}-$ node, and then propagates through the conducting system and through the muscle tissue. Because the propagation speed in the conducting system is two or three times higher than in the muscle [2], the propagating excitation wave is not symmetric but is elongated in the direction of conducting system. Because the excitable medium has the shape of ball layer with empty inner part, the most of cells in the square lattice are in the empty state during the whole simulation.

The transition rule that handle the evolution of the whole cellular automaton is composed of the following logical steps:

(i) the total number of all excited neighbours are counted and saved into the temporary variable named nb_excited,

(ii) during every $3^{\text {rd }} \mathrm{CA}$-step a muscle cell is excited with the probability of $50 \%$ if state $=0$ and nb_exited $>0$,

(iii) during every $3^{\text {rd }} \mathrm{CA}$-step the muscle cell with nonzero state value is decreased by one,

(iv) conducting system cell having state $=0$ and $n b_{-}$exited $>0$ is excited,

(v) $\mathrm{SA}$-node cell having state $=0$ is excited (self-excitation),

(vi) state value of the $\mathrm{SA}$-node cell having state $>0$ is decreased by one during every $10^{\text {th }} \mathrm{CA}$-step,

(vii) state value of the conducting system cell having state $>0$ is decreased by one during every $\mathrm{CA}$-step.

Temporary variable $n b_{-}$excited evaluated in the (i) step is used in the excitation steps (ii) and (iv). The steps from (ii) to (vii) are evaluated independently. The steps (ii) and (iii) operates during every $3^{\text {rd }}$ time step because it is assumed that propagation of the excitation front through the conducting system is three times higher than through the muscle tissue. The SA-node is independent of the rest of the excitable medium.

It has to be stressed out that a resting cell located next to the excitation wave front excite with probability of $50 \%$ (see step (ii)) if at least one of the neighbouring cells is excited. This leads to approximately isotropic propagation of the excitation wave front. If no probabilistic excitation of a cell is taken into account then anisotropic propagation of excitation waves occur that is caused by anisotropy of used lattice.

When inclusions - e.i. regions having slower excitability - are inserted then the morphology variable have to be extended by new state named inclusion. The morphology value of four is associated with an inclusion. The additional step should be added in the following form:

(viii) state value of a cell belonging to the inclusion having state $>0$ is decreased by one during every $9^{\text {th }} \mathrm{CA}$-step.

Propagation of excitation waves through the proposed topology, interactions of excitation waves with an excitatory disturbance, and interactions with an inclusion resulting in a response of such medium will be reported in the subsequent section. 


\section{Results and Discussion}

As mentioned before, the topology of the excitable medium used in the present $\mathrm{CA}$-model has the shape of the ball surface layer - several cells thick - with two simple arms of the conducting system and the pacemaker in between them, cf. Fig. 1. Such simplified topology serve as a toy-model that can helps to improve the understanding of the propagation, interactions and collisions of excitation waves, and interactions of those waves with excitatory disturbances and inclusions. Achieved results and experience can be used to construct models of the human heart.

\subsection{Propagation of Undistorted Excitation Waves in the Anisotropic Excitable Medium}

Excitation waves are generated - with given pacing rate - at the SA-node that is located at the top of the ball (cf. Fig. 1) and that operates as the pacemaker. The back propagation of excitation waves is protected due to non-excitability of cells having non-zero state variable, i.e. cells that are in any of the refractory states. Those excitation waves simple propagate through the excitable medium by excitation of cells in the resting state.
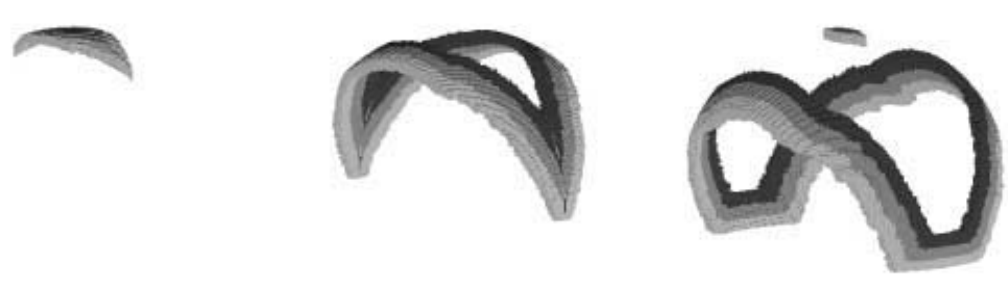

Fig. 2. A sequence of snapshots displaying the propagation of excitation waves through the anisotropic excitable medium created by the ball surface layer with the conducting system. The topology and orientation of the excitable medium are very same as in Fig. 1.

A sequence of excitation waves generated in the SA-node can be seen in Fig. 2 - generally sequences of figures start at the left-upper corner and continue in rows. From this figure, it is evident that propagation of the excitation wave front is anisotropic through given excitable medium. The excitation front propagates faster in the direction of arms of the conducting system as expected, compare to Fig. 1.

The excited state is associated with light grey colour. As the excitation of a given cell decreases, light grey colour becomes darker and then dark grey. 
The final resting state of this cell is depicted by the invisible colour. Therefore, excited cells laying in the back side of the surface layer of the ball are visible through those cells having invisible colour laying in the front layer, see Fig. 2.

The excitation wave propagates from the top of the ball to the bottom through the surface layer. Finally, two parts of the excitation front collide and disappear due to lack of cells in the resting state. One excitation wave is generated after the other and they propagate through the excitable medium. In this case, no interactions between different excitation waves occurs. The question is what will happen if waves collide in some more topologically complicated cases of the excitable medium - with inclusions having slower excitability then the rest of media - or when an excitatory disturbance is applied?

\subsection{Appearance of Spirals in the Excitable Medium with Initial Excitatory Disturbances}

Production of spirals in the excitable media is of the great interest. Spirals in the human heart are associated with dangerous and often deadly arrhythmias. Generally, spirals can be created in an excitable medium using a special initial configuration of cells and their initial excitation states, or using an excitable medium with an inhomogeneous propagation speed of the excitatory process. The attention is focused to the influence of the initial pre-excitation of selected parts of media in this subsection. It should be mentioned that the number of cells used in the simulations is changed - due to total simulation time - to value of $100^{3}$ from here to the end of the article.

The easiest way of the production of a spiral in the homogeneous and isotropic excitable medium is to excite, for example, a line of cells laying in the surface layer. In order to protect the propagation of the excitation waves up and down at the same time, upper left-half of the line of cells sitting next to the excited line is set into the refractory state, and at the same time, lower right-half of the line of cells sitting next to the excited line is set into the refractory state. The rest of the excitable medium - except the excited SA-node at the top - is set into the resting state. This leads to the propagation of one sole spiral having two arms through the excitable medium but this spiral spontaneously disappears after a certain period of time.

A another special initial pre-excitation of the excitable medium can be used to produce spirals, cf. Fig. 3. A line of cells laying in the surface layer is excited (dark grey) simultaneously with the SA-node. The line of cells sitting next to the excited line of cells on one side of the excited line is set into the refractory state (light grey). This produces two spirals in the excitable medium. Spirals overwhelms the activity of the SA-node and survive for ever as can be seen in Fig. 3. Please note that in this case, no special morphological disturbance or inhomogeneity of the excitable medium is necessary for initialization and surviving of the spiral.

A closer inspection of propagation of the excitation wave front in Fig. 3 gives the answer why spirals can survive for ever in this particular case. First, the excitation propagates from the $\mathrm{SA}$-node and from the excited line as can be 

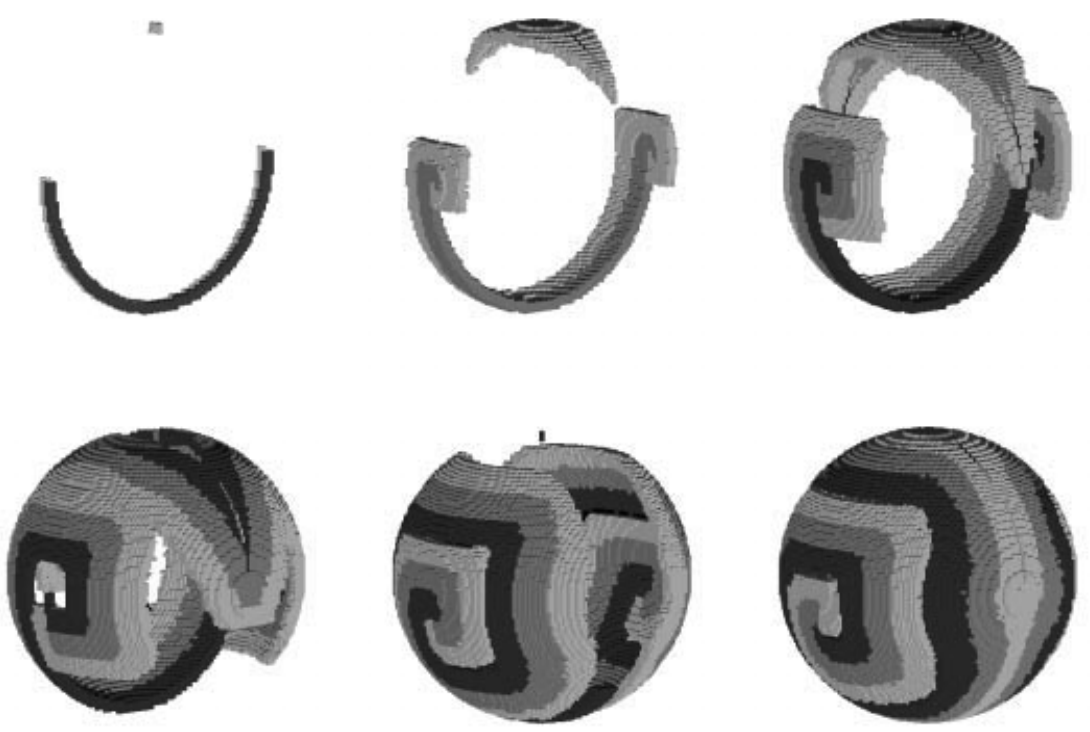

Fig. 3. A special initial pre-excitation of the medium is sufficient to produce spirals that can overwhelm the role of the SA-node. No special morphological disturbance of the excitable medium is necessary for initialization and surviving of the spiral.

seen in the first three figures. Two spirals having one arm are created at the ends of the initially pre-excited surface line. Consequently, on the first figure of the second row of figures, holes created by recovered cells and surrounded by cells in the refractory state - except a small gaps - appear around the ends of the originally excited line. Those holes are again invaded by few excited cells - from the above mentioned gaps - laying at the border of those holes. This activity overwhelms the pacemaking activity of the SA-node. From now, the centers of the spiral become the pacemakers.

From the above results, it is evident that spatio-temporal synchronization of excitation events and propagation of the excitatory process through the excitable medium with given topology plays the crucial role! Those observations cannot be neglected in any model of excitable media.

So far, we discussed the case where the SA-node is excited in synchrony with the pre-excited line of cells laying in the surface layer where the orientation of the conducting system - when we look from the top, i.e. from the position of the $\mathrm{SA}$-node - is perpendicular to this pre-excited line as can be seen from the Fig. 3. Spirals are present in this case. A natural question arise what happen in the case where the conducting system and the initially pre-excited line are parallel or when their mutual angle is different from those two positions?

For simplicity, assume that in the 'parallel' case the angle between the conducting system and the pre-excitation is zero. It is observed that in the 'parallel' 
case spirals are not produced. When the angle is increased above a certain orientation angle, spirals appear but they are not persistent. Above another critical orientation angle up to ninety degree all spirals become persistent.

\subsection{Spatio-Temporal Response of the Excitable Medium with Dependence on the Position and Size of Inclusions}

The last but not the least observation is related to inclusions of an elliptical region having slower excitability then the rest of the excitable media. An inclusion of such regions in the surface layer - having three times slower excitability then the rest of media - can leads to irregular/pathological spatio-temporal response of the system, cf. Fig. 4. The first figure in the upper row displays the topological position of the supercritical inclusion - i.e., excitatory irregularities are generated by it - related to the conducting system. Region is located near of the end of one arm of the conducting system.

When such inclusions are inserted and their size - depending on the ratio by which this region is slower - exceed some critical size then those inclusions produce excitations that back-propagates/spreads into the previously excited excitable medium - now again recovered - irrespectively of the conducting system. It seem that excitation 'tunnels' the natural barrier created by recovering medium when the simulations is observed. The excitatory process operates at time and space where under normal conditions without the inclusion cannot be present. The overall activity again depends on the relative position of the inclusion to the conducting system.

One another aspect of 'tunnelling' process should be mentioned here. For larger sizes of inclusions, a distorted excitation followed by abnormal excitation of the conducting system without excitation from the pacemaker is observed. In details, one observe the following: (i) excitation of the conducting system triggered by the pacemaker, (ii) normal excitation of the media to the moment when the inclusion is reached, (iii) 'tunnelling' of excitation through the inclusion, (iv) abnormal back-propagation of excitation that activates the conducting system without excitation from the pacemaker, and (v) finally, all irregular excitation activity tends to disappear when a new excitation sequence starts at the pacemaker.

All the previously discussed aspects of the excitable media - or their modifications - and many others should be observed in the more complicated simulations where the topology of the human heart is used. All become more complicated when the anisotropy of the media related of the muscle fiber is used even for the simplified ball topology. For the human heart the excitatory maps can be compared to the experimentally measured ones [2].

\section{Conclusion}

A cellular automaton model describing propagation and mutual interactions of excitation waves on the surface layer of a ball with fast accessory paths and 

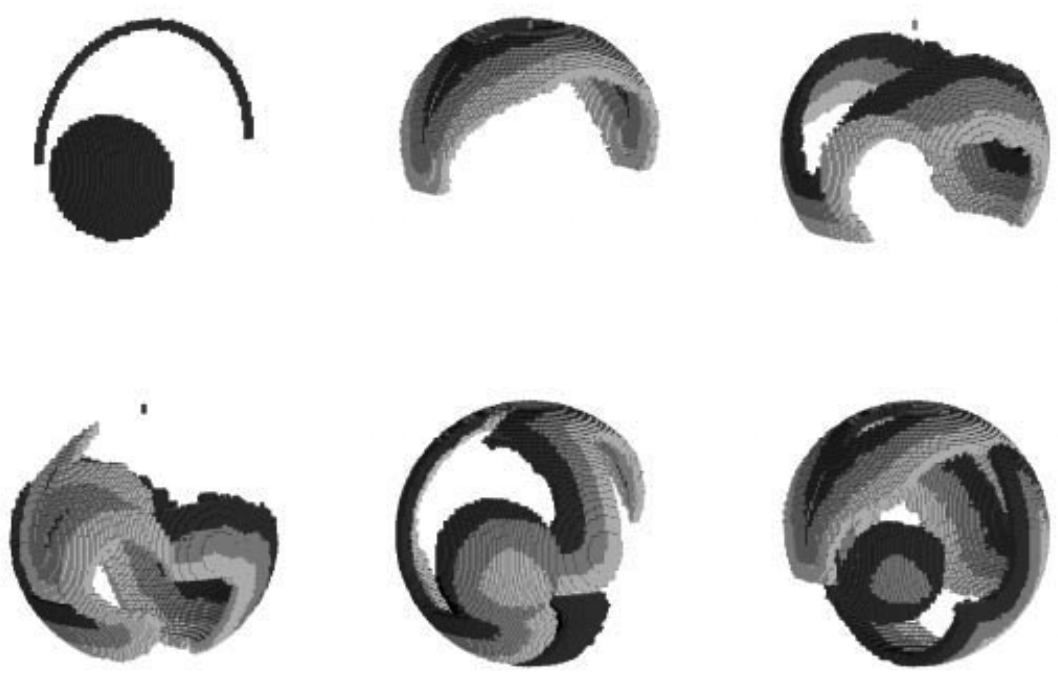

Fig. 4. Propagation of excitation in the surface layer with two different sizes of inclusion are showed in higher and lower rows. First figure displays the topology. The excitation invades the recovered medium in the third figure and distorts the subsequent evolution.

the pacemaker has been proposed. It is recognized that positions and sizes of excitatory disturbances and inclusions of the excitable medium are important and that they strongly influence the spatio-temporal response of this medium.

Propagation of undistorted excitation waves in the homogeneous excitable medium and appearance of spirals in it - induced by an applied initial excitatory disturbances, i.e. pre-excitations, are studied. Spirals do not appear for parallel orientation angle between the conducting system and the pre-excitation line. When the angle is increased above a certain orientation angle, spirals appear but they are not persistent. Above another critical orientation angle up to ninety degree all spirals become persistent.

The last but not the least observations are related to inclusions of elliptical regions having slower excitability then the rest of media. When such inclusions are inserted and their size - depending on the ratio by which this region is slower - exceeds some critical size then inclusions produce excitations that backpropagates into excitable medium irrespectively of the conducting system. The overall activity again depends on the relative position of the inclusion to the conducting system.

\section{Acknowledgments}

The author is greatly indebted to Prof. J.D. Eckart for his publicly available $\mathrm{CA}$-environment called Cellular/Cellang [3] that have been used to compute 
greater part of the presented results. The author acknowledge financial support from the Czech Ministry of Education under project LN00B084.

\section{References}

1. Chopard, B., Droz, M.: Cellular Automata Modeling of Physical Systems. Cambridge University Press, Cambridge, 1998

2. Durrer, D., van Dam, R. Th., Freud, G.E., Janse, M.J., Meijler, F.L. and Arzbaecher, R.C.: Total Excitation of the Isolated Human Heart. Circulation 41 (1970) 899-912

3. Eckart, J.D.: The Cellular Automata Simulation System: Cellular/Cellang. (19911999) http://www.cs.runet.edu/ dana/

4. Fast, V.G. and Efimov I.R.: Stability of vortex rotation in an excitable cellular medium. Physica D 49 (1991) 75-81

5. Fast, V.G., Efimov I.R. and Krinsky, V.I.: Transition from circular to linear rotation of a vortex in an excitable cellular medium. Physics Letters A 151 (1990) $157-161$

6. Warwick, R. and Williams, P.L.: Gray's Anatomy - 35th edition. Longman, Edinburgh, 1973

7. Guyton, A.C. and Hall, J.E.: Textbook of Medical Physiology. W.B. Saunders Company, Philadephia, London, Toronto, Montreal, Sydney, Tokyo, 1996

8. Hulín, I. et al.: Patofyziológia. Slovak Academic Press, Bratislava, 1998

9. Graphical library: OpenGL API. Silicon Graphics, Inc., California, USA, (19932002) http://www.sun.com/software/opengl/

10. Toffoli, T.: Cellular automata as an alternative to (rather than an approximation of) differential equations in modelling physics. Physica 10D (1984) 117-127

11. Toffoli, T. and Margolus, N.: Cellular Automata Theory, The MIT Press, Cambridge, Massachusetts, London, England, 1987

12. Vichniac, G.Y.: Simulation physics with cellular automata, Physic 10D (1984) 96-116

13. Weimar, J.R.: Simulation with Cellular Automata, Logos Verlag, Berlin, 1997

14. Wiener, N. and Rosenblueth, A.: The mathematical formulation of the problem of conduction of impulses in a network of connected excitable elements, specifically in cardiac muscle. Arch. Inst. Cardiol. Mexico 16 (1946) 205-265 